\title{
INFRARED OBSERVATIONS OF CIRCUMSTELLAR MOLECULES
}

\author{
Albert Betz \\ Space Sciences Laboratory \\ University of California \\ Berkeley, CA 94720 USA
}

\section{Introduction}

This review presents an updated listing of infrared observations of circumstellar molecules. The compendium is restricted to the more abundant species with infrared spectra accessible to groundbased observations. No analysis is offered on the chemical importance of a particular species, and no attempt is made to correlate the infrared work with the much larger body of data available from radio frequency observations. Some discussions of these topics may be found in the more comprehensive reviews listed below. The observations described here include both the successful and the unrewarded efforts. Often the negative results from attempted observations are not widely disseminated, even though such results can sometimes be of significant theoretical value. Discussions of infrared stellar and circumstellar spectroscopy from other vantage points may be found in the following reviews. In 1979 Merrill and Ridgway surveyed the contributions of infrared spectroscopy to studies of stellar photospheres and the near circumstellar environment. The following year Zuckerman (1980) summarized the current state of knowledge on circumstellar molecular clouds with attention given to both infrared and microwave observations. Ridgway and Keady (1981) subsequently noted the importance of infrared spectroscopy for studies of circumstellar gas and dust in the star IRC +10216 . In 1983 Hinkle reviewed the high-resolution spectroscopy of late-type circumstellar shells in general but emphasized chemistry and kinematics. Finally, in the proceedings of the 1984 UCLA meeting on mass-loss, Omont (1985) reviewed the physical and chemical structure of circumstellar envelopes and IRC +10216 in particular, and Wannier (1985) discussed the significance of infrared spectroscopy for studies of mass-loss in latetype stars.

The observations listed below have been done with three different types of high resolution spectrometers. Details on the operation of specific instruments may be found in the presentations by Lacy (1979) on Fabry-Perot spectroscopy, by Hall and coworkers (1978a) on Fourier transform spectroscopy (FTS), and by Betz (1981a) on laser heterodyne spectroscopy. These instruments may be regarded as both competitive and complementary; the difference lies entirely in the problem at hand. One point worth noting is that grating spectrometers, which are generally preferred at optical wavelengths, are generally disfavored for high resolution infrared spectroscopy. This bias may change in the future, however, with the advent of cooled infrared echelles coupled to sensitive array detectors.

\section{Principal Detected Species}

$\mathrm{CO}$

Carbon monoxide was the first molecule detected in circumstellar sources at infrared wavelengths. In 1973, Geballe, Wollman, and Rank reported the detection of ${ }^{12} \mathrm{CO}$ and ${ }^{13} \mathrm{CO}$ in IRC +10216, VY CMa, and NML Cyg. Further observations of isotopic CO were discussed in a 
subsequent paper by Rank, Geballe, and Wollman (1974). This was the first time that the $4.7 \mu \mathrm{m}$ band of $\mathrm{CO}$ had been observed in stars with enough resolution to separate the individual vibration-rotation lines. The observations were done with a high resolution $\left(0.15 \mathrm{~cm}^{-1}\right)$ scanning Fabry-Perot spectrometer on the 3-m Shane telescope at Lick Observatory. Synthetic spectra were calculated to fit the data from IRC +10216 . The best fits were achieved with a rotational temperature of $400 \mathrm{~K}$, a ${ }^{12} \mathrm{CO}$ column density of $5 \times 10^{19} \mathrm{~cm}^{-2}$, and an (assumed) ${ }^{12} \mathrm{CO} /{ }^{13} \mathrm{CO}$ ratio of 8 . In this analysis a larger isotopic ratio leads to a correspondingly larger value for the $\mathrm{CO}$ column density.

In 1977 Barnes, Beer, Hinkle, and Lambert published more extensive infrared observations of $\mathrm{CO}$ in IRC +10216 . From an analysis of $3-5 \mu \mathrm{m}$ spectra taken at a resolution of $0.15 \mathrm{~cm}^{-1}$ with the JPL FTS on the McDonald 2.7-m telescope, these authors derived an excitation temperature of $400 \mathrm{~K}$ and a ${ }^{12} \mathrm{CO}$ column density of $\sim 2 \times 10^{20} \mathrm{~cm}^{-2}$. The isotopic ratios were estimated to be: ${ }^{12} \mathrm{C} /{ }^{13} \mathrm{C} \approx 20,{ }^{16} \mathrm{O} /{ }^{17} \mathrm{O} \approx 1600$, and ${ }^{17} \mathrm{O} /{ }^{18} \mathrm{O} \approx 1$.

More recent infrared observations of $\mathrm{CO}$ have used the molecule as a tracer of dynamical activity in circumstellar gas. For example, Ridgway and Hall (1980) used the KPNO FTS to observe a shift in center velocities of (2-0) $\mathrm{CO}$ lines as a function of excitation energy in IRC +10216 and several similar objects. Keady (1982) has analyzed an extensive set of $4.6 \mu \mathrm{m}(1-0)$ and $2.3 \mu \mathrm{m}(2-0) \mathrm{CO}$ line profiles from IRC +10216 to produce a model for the excitation and distribution of gas and dust within $\mathbf{1 0 0}$ radii of the central star. Infrared CO lines have also been seen in regions of star formation. Hall and coworkers (1978b) detected multiple absorption components in the $4.6 \mu \mathrm{m}$ fundamental band of $\mathrm{CO}$ in the $\mathrm{BN}$ source in Orion. Most of this CO, however, is thought to reside in the foreground quiescent gas of the Orion molecular cloud and in the expanding component from the plateau source, and hence is not strictly circumstellar.

\section{$\mathrm{C}_{2} \mathrm{H}_{2}$}

Acetylene, $\mathrm{C}_{2} \mathrm{H}_{2}$, was first detected in IRC +10216 by Ridgway and coworkers (1976), who observed a blend of Q-branch lines in the $\nu_{1}+\nu_{5}$ band at $4091 \mathrm{~cm}^{-1}$. A rotational temperature of 200-300 $\mathrm{K}$ was deduced from the drop-off in line strength near $\mathrm{J}=16$, which suggested a circumstellar locale. Using an average temperature of $250 \mathrm{~K}$ and the published band strength available at the time, the authors derived a $\mathrm{C}_{2} \mathrm{H}_{2}$ abundance of $3 \times 10^{19} \mathrm{~cm}^{-2}$, which was about $14 \%$ of the estimated $\mathrm{CO}$ abundance.

Two years later, Ridgway, Carbon, and Hall (1978) detected acetylene in a variety of carbon-rich stars. Observations of the $3-\mu \mathrm{m} \nu_{3}$ band of acetylene were done using an FTS spectrometer on the Mayall 4-m telescope. In most stars the lines appeared to be photospheric in origin with excitation temperatures near $2000 \mathrm{~K}$. In IRC +10216 , however, the lines seemed to be circumstellar. Numerous lines from both ${ }^{12} \mathrm{C}_{2} \mathrm{H}_{2}$ and ${ }^{12} \mathrm{CH}^{13} \mathrm{CH}$ were observed, and a rotational temperature of $\sim 400-800 \mathrm{~K}$ was estimated from the excitation of the strongest lines. In addition, the strong lines also showed P-Cygni emission which had not been seen before, probably for instrumental reasons. Compared to the earlier $2.4 \mu \mathrm{m}$ effort, the $3.1 \mu \mathrm{m}$ observations of acetylene were better able to penetrate the dust obscuration and thereby probe warmer regions of the circumstellar envelope.

In 1982 Rinsland, Baldacci, and Rao measured the integrated strength of the Q-branch of the $\nu_{1}+\nu_{5}$ acetylene band in the laboratory. Their result of $1.67 \mathrm{~cm}^{-2} \mathrm{~atm}^{-1}$ at $300 \mathrm{~K}$ was a factor of 12 higher than the laboratory value used by Ridgway and coworkers in 1976 to interpret the $2.4 \mu \mathrm{m}$ acetylene data from IRC +10216 . Consequently, Rinsland, Baldacci, and Rao suggested that the abundance of acetylene in IRC +10216 should be revised downwards to $3 \pm 1 \times 10^{18} \mathrm{~cm}^{-2}$.

Knowledge of the $\mathrm{C}_{2} \mathrm{H}_{2}$ abundance in IRC +10216 is important for testing photoproduction models of radical species such as $\mathrm{C}_{2} \mathrm{H}$. In the photodissociation model of Huggins, Glassgold, and Morris (1984), $\mathrm{C}_{2} \mathrm{H}_{2}$ is the main source for any photogenerated $\mathrm{C}_{2} \mathrm{H}$ in the outer envelope. The high abundance of $\mathrm{C}_{2} \mathrm{H}_{2}$ originally estimated by Ridgway et al. (1976) leads to a stronger $\mathrm{C}_{2} \mathrm{H}$ line at $87 \mathrm{GHz}$ than is actually observed, if $\mathrm{C}_{2} \mathrm{H}$ has a photochemical origin. The revised abundance for $\mathrm{C}_{2} \mathrm{H}_{2}$ suggested by Rinsland, Baldacci, and Rao is more consistent with the lower abundance 
of $\mathrm{C}_{2} \mathrm{H}$ that is observed. However, a non-photochemical origin for $\mathrm{C}_{2} \mathrm{H}$ is not necessarily ruled out by these results. On the other hand, a higher $\mathrm{C}_{2} \mathrm{H}_{2}$ abundance would require modification of the photochemical model.

Acetylene also has a strong $\nu_{5}$ vibration-rotation band centered near $730 \mathrm{~cm}^{-1}$. Keady and Ridgway have observed a number of ${ }^{12} \mathrm{C}_{2} \mathrm{H}_{2}$ and ${ }^{13} \mathrm{CH}^{12} \mathrm{CH} \nu_{5}$ ro-vibrational lines in broadband FTS spectra of IRC +10216. Only $\mathrm{R}$-branch lines between $\mathrm{J}=\mathbf{2 9 - 4 5}$ were seen; the lower-J lines were obscured by atmospheric absorption and the cutoff of a bandpass filter. The observed lines have a circumstellar expansion velocity of $\sim 11 \mathrm{~km} / \mathrm{s}$, which implies that the gas is not fully accelerated to the $14 \mathrm{~km} / \mathrm{s}$ terminal velocity. Many of the stronger lines also showed P-Cygni type emission. The new data suggest that the circumstellar abundance of acetylene is high, $\sim 2 \times 10^{19} \mathrm{~cm}^{-2}$ in the observed region. This acetylene, however, is closer to the star than the gas seen in "cooler" lines at $3 \mu \mathrm{m}$ (Keady 1985). Kostiuk and coworkers (1985) have also observed lines in the $\nu_{5}$ acetylene band, but at the much higher resolution $(0.3 \mathrm{~km} / \mathrm{s})$ afforded by laser heterodyne spectroscopy. Heterodyne observations can be especially useful for measuring the acceleration of circumstellar gas in regions close to the photosphere.

Molecules observed in the infrared are usually seen in absorption, and column densities that are measured can be affected by optical depth effects, both in the continuum and in the line. Especially for optically-thin molecules with uniform mixing ratios, the inferred abundance will be wavelength dependent because the column length extends to the (wavelength dependent) radius where the dust becomes optically thick. Omont (1985) has discussed the related problem of comparing abundances deduced from emission lines (at radio frequencies) with those derived from absorption (in the infrared).

\section{HCN}

The first infrared detection of circumstellar HCN was announced in 1978, about 7 years after the first microwave detection of $\mathrm{HCN}$ in the same source--IRC +10216. Ridgway, Carbon, and Hall (1978) detected a variety of $3 \mu \mathrm{m}$ HCN absorption bands in a number of carbon-rich stars. In most sources the HCN appears to be distinctly photospheric. In IRC +10216 , however, the intensities of the strongest lines indicate a cooler excitation temperature of $400-800 \mathrm{~K}$, similar to that observed for $\mathrm{C}_{2} \mathrm{H}_{2}$ lines in the same spectral region. No abundance estimate was explicitly stated at the time, but a value $\geq 1.5 \times 10^{18} \mathrm{~cm}^{-2}$ was subsequently presented (Hall and Ridgway 1978). The estimate was highly uncertain, however, because lines in the HCN $\nu_{3}$ fundamental band were highly saturated, and.laboratory measurements of the strengths of the combination bands were relatively crude at the time.

$\mathrm{SiO}$

The first infrared detection of $\mathrm{SiO}$ in distinctly circumstellar gas did not occur until 1978 . Previously, infrared observations of $\mathrm{SiO}$ had been limited to the $4 \mu \mathrm{m}$ overtone bands in the photospheric spectra of evolved giant and supergiant stars. In 1978 Geballe, Lacy, and Beck (1979) used a Fabry-Perot spectrometer to detect a number of SiO absorption lines in the star VY CMa. The lines, originating in the 1-0 fundamental band at $8.3 \mu \mathrm{m}$, indicated an excitation temperature $\sim 600 \mathrm{~K}$ and a column density of $7 \pm 3 \times 10^{17} \mathrm{~cm}^{-2}$. This column density together with an $\mathrm{H}_{2}$ density derived from $\mathrm{H}_{2} \mathrm{O}$ maser observations suggested an $[\mathrm{SiO}] /[\mathrm{H}]$ abundance ratio $\sim 10^{-6}$, which was about 30 times less than the solar $[\mathrm{Si}] /[\mathrm{H}]$ ratio. The authors concluded that most of the silicon is locked away in dust grains and not observable in gaseous form. The observations also indicated that the isotopic ratios for ${ }^{28} \mathrm{Si},{ }^{29} \mathrm{Si}$, and ${ }^{30} \mathrm{Si}$ were roughly terrestrial. The stronger ${ }^{28} \mathrm{SiO}$ lines showed P-Cygni emission, which was further evidence that the circumstellar gas was still rather close to the star. The authors estimated the emission region to be $\sim 0.15$ arcsec, which corresponds to $1 \times 10^{15} \mathrm{~cm}$ if a distance of $\sim 1.5 \mathrm{kpc}$ to VY CMa is adopted.

In IRC +10216 absorption from the $\mathrm{SiO}$ fundamental at $1200 \mathrm{~cm}^{-1}(8 \mu \mathrm{m})$ has not been detected, even though $\mathrm{SiO}$ is seen at microwave frequencies to have a beam-averaged column density of $\sim 10^{16} \mathrm{~cm}^{-2}$. The infrared limit is $<10^{17} \mathrm{~cm}^{-2}$, but the measurement is weighted toward gas much closer to the star (Keady 1985). SiS similarly is not seen in the infrared, although for 
different reasons. The SiS vibration fundamental is centered around $749 \mathrm{~cm}^{-1}(\mathbf{1 3 . 4} \mu \mathrm{m})$ and is difficult to observe because of absorption from atmospheric $\mathrm{CO}_{2}$.

\section{Trace species}

$\mathrm{NH}_{3}$

In 1978 ammonia was first detected in circumstellar gas, although it was known to be a constituent in interstellar clouds for over a decade. Several $\mathrm{NH}_{3}$ absorption lines in the $\nu_{2}$ band at $10.5 \mu \mathrm{m}$ were observed in the carbon-rich source IRC +10216 with the new technique of infrared heterodyne spectroscopy (Betz, McLaren, and Spears 1979; Betz and McLaren 1980). Shortly thereafter $\mathrm{NH}_{3}$ lines were also seen in a number of oxygen-rich supergiant stars (McLaren and Betz 1980). Generally a single rotational temperature does not adequately indicate the excitation of all observed lines. Absorption lines from low-J levels $(\mathrm{J}=\mathbf{0 - 4})$ have rotational temperatures near 50-100 K, whereas the high-J lines $(\mathrm{J}=6-9)$ are better fit with values closer to $300-500 \mathrm{~K}$. In all cases, however, the column densities are high, typically $10^{16}-10^{17} \mathrm{~cm}^{-2}$, which is several orders of magnitude higher than the abundances predicted by photospheric freeze-out models. In fact, in o-Ceti the lines appear saturated, and the distinction between photospheric and circumstellar gas may be blurred (Betz and Goldhaber 1985).

$\mathrm{CH}_{4}$

Methane, $\mathrm{CH}_{4}$, was first detected (once again) in IRC +10216 by Hall and Ridgway (1978). Their FTS observations of low excitation lines in the $\nu_{3}$ band near $3.3 \mu$ m were interpreted with a $300 \mathrm{~K}$ excitation temperature to deduce a column density of $2.5 \pm 1.0 \times 10^{17} \mathrm{~cm}^{-2}$. Subsequently, Clegg, Hinkle, and Lambert (1982) observed 20 absorption lines in the $\nu_{3}$ band of methane in $\mathbb{R C}+10216$. From the data the authors derived a rotational temperature of $162 \pm 24$ $\mathrm{K}$ and a column density of $1.8 \times 10^{16} \mathrm{~cm}^{-2}$. This abundance is about an order of magnitude less than the preliminary estimate of Hall and Ridgway (1978). The difference was partially attributed to the lower (but more accurate) estimate for the rotational temperature which reduced the partition function. Because methane was only seen in cool gas at a terminal velocity of $\sim 14$ $\mathrm{km} / \mathrm{s}$ relative to the star, the authors suggested that the net production of methane might be enhanced below a critical temperature by gas-carbon grain reactions.

$\mathrm{SiH}_{4}$

Silane is the silicon analog of methane. It was first detected in IRC +10216 in 1983 from absorption lines in the strong $\nu_{4}$ band at $11 \mu \mathrm{m}$ (Goldhaber and Betz 1984). From the 13 line profiles observed at high resolution $(0.2 \mathrm{~km} / \mathrm{s})$ with a heterodyne spectrometer, a rather welldefined rotational temperature of $173 \pm 27 \mathrm{~K}$ was deduced along with a column density of $2 \times 10^{15} \mathrm{~cm}^{-2}$. The observed abundance is significantly higher than the estimate inferred from freeze-out models with photospheric pressures of 100 dynes $\mathrm{cm}^{-2}$ (McCabe, Smith, and Clegg 1979).

Independent observations of silane by Keady and Ridgway (1986) lead to similar conclusions. However, their wideband FTS spectrum of IRC +10216 (taken in 1983) covers a much broader spectral range than the heterodyne data, and gives a more complete picture of the stronger silane lines near $11 \mu \mathrm{m}$. From their analysis the rotational excitation of silane varies from $100 \mathrm{~K}$ to $200 \mathrm{~K}$, and the $\left[\mathrm{SiH}_{4}\right] /\left[\mathrm{H}_{2}\right]$ abundance ratio appears to be $2 \times 10^{-7}$. For a mass-loss rate of $2 \times 10^{-5} \mathrm{M}_{\odot} / \mathrm{yr}$ (Keady, Hall, and Ridgway 1986), the silane column density is then $4 \times 10^{15} \mathrm{~cm}^{-2}$. All the silane lines appear at the terminal velocity of $14 \mathrm{~km} / \mathrm{s}$. A model calculation for such cool gas suggests that silane does not exist closer to the star than 30 stellar radii. Consequently, circumstellar reaction sites such as grain surfaces are needed to produce the observed gas.

Silane and methane are tetrahedral molecules without permanent dipole moments. Rotational transitions within a vibrational state are weakly allowed, however, for high-J levels because 
of centrifugal distortion. Under the relatively low density conditions extant in circumstellar envelopes, excitation of both molecules is essentially governed by the radiation field and not by collisions. Rotational temperatures deduced from the infrared data may be more a measure of the infrared radiation field than the actual kinetic temperature of the gas.

A preliminary search for silane in the OH/IR supergiants NML Cygnus and VY CMa failed to reveal any lines (Goldhaber and Betz 1985, unpublished). A more thorough search still needs to be done, however, before definite conclusions can be drawn. Both of these stars have strong SiO masers at microwave frequencies and strong $\mathrm{SiO}$ absorption lines in the infrared. The fact that $\mathrm{SiH}_{4}$ is not easily seen suggests that silicon may be locked up in $\mathrm{SiO}$ in these oxygen-rich stars. Silane oxidizes rapidly in the presence of oxygen in a highly exothermic reaction. Consequently, the equilibrium abundance for silane in an oxygen-rich stellar atmosphere is small. The fact that silane, methane, and ammonia are all observed to be abundant and concentrated at the terminal velocity for ejected circumstellar material suggests that the formation of these saturated-bond hydrides may be catalytically enhanced by grain-surface reactions, at least in carbon-rich stars. More observations of silane in both carbon- and oxygen-rich stars is needed, however, before any definite conclusions can be drawn. Recently, Schrey and coworkers (1985) reported some additional heterodyne observations of silane in IRC +10216 .

$\mathrm{C}_{2} \mathrm{H}_{4}$

Ethylene, $\mathrm{C}_{2} \mathrm{H}_{4}$, was first detected in 1981 in the carbon star IRC +10216 (Betz 1981b). Because ethylene has a symmetric structure and no permanent dipole moment, rotational transitions generally do not exist, and only lines in the infrared vibration-rotation bands are observable. Two weak lines in the $\nu_{7}$ vibrational band at $950 \mathrm{~cm}^{-1}(10.5 \mu \mathrm{m})$ were detected with heterodyne techniques and used to deduce a rotational temperature $>300 \mathrm{~K}$ and a column density of $\sim 10^{16} \mathrm{~cm}^{-2}$. The possibility that the lines could be partially filled in by continuum emission from warm dust suggested that a higher abundance up to $10^{17} \mathrm{~cm}^{-2}$ might also be possible. However, no detailed modeling to judge the magnitude of this effect was done at the time. As is the case for most circumstellar absorption lines in IRC +10216 , the ethylene lines were observed at a velocity blue-shifted $14 \mathrm{~km} / \mathrm{s}$ from the stellar systematic velocity of $-26 \mathrm{~km} / \mathrm{s} \mathrm{V}_{\mathrm{LSR}}$.

More recent observations of IRC $+\mathbf{1 0 2 1 6}$ by Goldhaber, Betz, and Ottusch (1986) confirm the earlier identification but place more quantitative limits on the temperature, abundance, and distribution of ethylene. From observations of 6 lines in the $\nu_{7}$ band, it is now clear that ethylene, unlike methane or silane, cannot be described by a single rotational temperature. Although an average temperature of $100 \mathrm{~K}$ can be fitted to the observed lines, intensities of the low-J lines $(\mathrm{J}<10)$ are better modeled by temperatures near $50 \mathrm{~K}$, whereas the high-J lines $(\mathrm{J}=10-20)$ are better fitted by values around $\sim 300 \mathrm{~K}$.

Interpretation of the new data is bolstered by new laboratory measurements of the individual line strengths for the observed lines. Previously line strengths were calculated from a measured band strength because individual measurements were not available. Such a calculation can be subject to errors from unconsidered effects such as interband coriolis coupling which can perturb the strengths of various lines. However, for ethylene, the measured intensities for the observed lines did not vary significantly from the values calculated from the band strength.

The column density estimated from the new data is $4 \times 10^{15} \mathrm{~cm}^{-2}$. This abundance estimate is not especially sensitive to temperature, as long as a fit is made to all the observed lines. Actually, the estimate should be taken as a lower limit since no consideration was given to possible line-filling by continuum radiation from commingled warm dust. In order to determine the full extent of any such line-filling, however, one would need a detailed model of the line forming region in the circumstellar atmosphere. It should be remembered that radiation transfer models for circumstellar envelopes generally predict that the dust is significantly hotter than the gas at a given distance from the star. 
$\mathrm{C}_{2} \mathrm{H}$

Chemical equilibrium calculations for the production of molecules in the carbon-rich photosphere of IRC +10216 do not predict a large abundance for radical species such as $\mathrm{C}_{2} \mathrm{H}(\mathrm{McCabe}$, Smith, and Clegg 1979). On the other hand, photochemical models predict that $\mathrm{C}_{2} \mathrm{H}$ can be produced efficiently by UV-dissociation of $\mathrm{C}_{2} \mathrm{H}_{2}$ in the outer circumstellar envelope (Huggins, Glassgold, and Morris 1984). The $\mathrm{N}=1-0$ rotational multiplet of $\mathrm{C}_{2} \mathrm{H}$ has been detected at 87 $\mathrm{GHz}$ in IRC +10216, but one line (even with hyperfine structure) is not enough to deduce both the excitation and the abundance of this species. $\mathrm{C}_{2} \mathrm{H}$ also has reasonably strong electronic bands at 4010 and $4108 \mathrm{~cm}^{-1}(2.4 \mu \mathrm{m})$. Keady and Hinkle (1986) recently have reported the detection of these bands in high resolution spectra of IRC +10216 . The dozen or so lines observed indicate a very low but well determined excitation temperature of $12.5 \pm 1.5 \mathrm{~K}$. No absorption is seen from rotational levels with $\mathrm{J}>4$. Absorption occurs only at the $14 \mathrm{~km} / \mathrm{s}$ terminal velocity. There is no low velocity component such as that seen in some $\mathrm{CO}$ and $\mathrm{C}_{2} \mathrm{H}_{2}$ lines which would indicate incompletely accelerated gas closer to the star. Consequently, Keady and Hinkle (1986) conclude that the $\mathrm{C}_{2} \mathrm{H}$ detected at $2.4 \mu \mathrm{m}$ is undoubtedly produced in the outer envelope by photochemical reactions.

CS

CS is another molecule that shows up in the KPNO FTS spectrum of IRC +10216 . In an interval between $1230 \mathrm{~cm}^{-1}$ and $1180 \mathrm{~cm}^{-1}(8.1-8.5 \mu \mathrm{m})$ where the atmosphere is transparent, the P10 through P44 lines of the CS rotation-vibration band have been observed (Keady 1985). The $\mathrm{R}$-branch lines at higher frequencies are blocked from view by atmospheric absorption. The P10 line appears saturated at the $14 \mathrm{~km} / \mathrm{s}$ terminal velocity for circumstellar gas in IRC +10216 . High-J lines like the P44 line, on the other hand, have a lower expansion velocity of $11 \mathrm{~km} / \mathrm{s}$ and show P-Cygni type emission. This behavior is similar to that seen in high excitation lines of CO and $\mathrm{C}_{2} \mathrm{H}_{2}$ which reveal hot gas not fully accelerated to the terminal velocity. The infrared data indicate that $\mathrm{CS}$ is relatively abundant in the inner envelope of $\mathrm{IRC}+10216$. A $[\mathrm{CS}] /\left[\mathrm{H}_{2}\right]$ abundance ratio of $2 \times 10^{-4}$ is needed to model the observed line profiles (Keady 1985). This fractional abundance is significantly higher than the $2 \times 10^{-7}$ estimate derived from radio observations, which preferentially measure gas in the outer circumstellar envelope.

\section{Undetected Species}

$\mathrm{H}_{2}$

Vibrational emission at $2.1 \mu \mathrm{m}$ from shock-excited $\mathrm{H}_{2}$ has been directly observed in planetary nebulae and some protostellar sources (see Beckwith 1981, and references therein), but at present there is no direct observational evidence for $\mathrm{H}_{2}$ in thermally-excited circumstellar gas. The presence of $\mathrm{H}_{2}$ as the major component of mass-loss is generally inferred from the excitation of other observable species. Of course, $\mathrm{H}_{2}$ absorption lines have been seen in the ultraviolet spectra of many stars, but these lines are from foreground interstellar gas. Several attempts have been made to detect the infrared quadrupole lines of circumstellar $\mathrm{H}_{2}$ in $\mathrm{IRC}+10216$, but so far all results have been negative. Nevertheless, the upper limits to the $\mathrm{H}_{2}$ abundance deduced from these observations are now low enough to be theoretically interesting.

In 1984 Lacy and Serabyn used a Fabry-Perot spectrometer with a resolution of $0.02 \mathrm{~cm}^{-1}$ at the IRTF on Mauna Kea in an attempt to detect the S(2) pure rotational line of molecular hydrogen at $12.2 \mu \mathrm{m}$ (Lacy 1985). Although a line was detected at the expected frequency, a careful analysis showed that interference from a hot-band transition of acetylene $\left(\mathrm{C}_{2} \mathrm{H}_{2}\right)$ could produce the observed spectrum. From the intensities of nearby acetylene lines observed at KPNO by Ridgway, and an adopted mass-loss rate of $10^{-4} \mathrm{M}_{\odot} / \mathrm{yr}$, the intensity of the $\mathrm{C}_{2} \mathrm{H}_{2}$ line at $12.2 \mu \mathrm{m}$ was calculated to be about 10 times the expected intensity of the $\mathrm{H}_{2}$ line. Careful modeling of the $\mathrm{C}_{2} \mathrm{H}_{2}$ line profile would be needed to determine any residual absorption due to $\mathrm{H}_{2}$. Interference from acetylene with the $\mathrm{S}(2)$ line of molecular hydrogen can therefore be expected for any 
carbon-rich source. Another observational problem with this spectral interval is that it is very close to a water vapor line which hampers observations at low altitude groundbased sites. Consequently, because of these systematic problems, searching for the $S(2)$ pure rotational line may not be a good way to detect $\mathrm{H}_{2}$ in circumstellar sources. The $\mathrm{S}(1)$ rotational line at $17 \mu \mathrm{m}$ may be a better choice, but it too is close to water lines.

In FTS observations with the 4-m Mayall Telescope at Kitt Peak, IRC + 10216 was observed in 1979 at $\mathrm{K}$-band wavelengths in an attempt to detect the $\mathrm{S}(1)$ vibration-rotation line of $\mathrm{H}_{2}$ at $2.1 \mu \mathrm{m}$. The results of a 10-hour integration produced no clear detection but did set an upper limit of $<4 \times 10^{-5} \mathrm{M}_{\odot} / \mathrm{yr}$ for the mass-loss rate of IRC +10216 (Keady 1986). The result is not very sensitive to the assumed excitation temperature for $\mathrm{H}_{2}$, and it measures only to a depth where the $2 \mu \mathrm{m}$ continuum becomes optically thick. This upper limit is currently the best direct estimate for the $\mathrm{H}_{2}$ abundance, and it is only slightly higher than the mass-loss rate inferred indirectly from model calculations of infrared absorption and microwave emission lines of $\mathrm{CO}$.

\section{$\mathrm{CH}, \mathrm{NH}$, and $\mathrm{OH}$}

In late-type stars there are many simple molecules which are abundant in photospheres but not evident in cooler circumstellar gas. Presumably the difference lies in the chemistry of the initially ejected material. For example, the infrared vibration-rotation lines of $\mathrm{CH}, \mathrm{NH}$, and $\mathrm{OH}$ have yet to be conclusively identified in cool circumstellar gas, even though they are readily detected in 3-4 $\mu \mathrm{m}$ spectra of warmer photospheric gas. Of course, $\mathrm{OH}$ is known from its maser lines to be a significant constituent in the extended shells of oxygen-rich supergiants, but this gas is presumably formed by the UV-photodissociation of $\mathrm{H}_{2} \mathrm{O}$.

$\mathrm{CN}$

$\mathrm{CN}$ has a vibration-rotation band at $4.9 \mu \mathrm{m}$ and electronic bands near $2 \mu \mathrm{m}$. Infrared lines from the short-wavelength bands can be quite strong in the photospheres of carbon-rich stars, but no such lines are seen originating from cooler circumstellar material. Wootten et al. (1982) derived an upper limit of $2 \times 10^{16} \mathrm{~cm}^{-2}$ for the circumstellar CN abundance in IRC +10216 from a $4.9 \mu \mathrm{m}$ FTS spectrum. Keady (1985) also reports a negative infrared result on CN. The problem with the $4.9 \mu \mathrm{m}$ band is that some $\mathrm{CN}$ lines are obscured by atmospheric absorption. Of course, $\mathrm{CN}$ emission at microwave frequencies has already been detected in IRC +10216 . Wootten et al. (1982) used the intensity of the $227 \mathrm{GHz} \mathrm{N}=2-1$ line and an adopted excitation temperature of $\sim 16 \mathrm{~K}$ to estimate the $\mathrm{CN}$ column density at $1.7 \times 10^{15} \mathrm{~cm}^{-2}$. The $\mathrm{CN}$ responsible for the microwave emission is thought to originate in the outer envelope from the photodissociation of HCN by interstellar-UV radiation.

$\mathrm{HCl}$

In 1982 Clegg, Hinkle, and Lambert reported a 1980 search for hydrogen chloride in IRC +10216. Absorption lines in the $3 \mu \mathrm{m} \nu_{3}$ band were expected to be observable with the KPNO FTS provided that most of the $\mathrm{Cl}$ was associated into $\mathrm{HCl}$. No lines were in fact detected, and the upper limit to the $\mathrm{HCl}$ abundance was estimated to be $2.3 \times 10^{15} \mathrm{~cm}^{-2}$. This limit is a factor of 10 to 20 less than the predicted abundance, and suggests that $\mathrm{Cl}$ may reside in either another gaseous species such as $\mathrm{CH}_{3} \mathrm{Cl}$ or in a condensate such as $\mathrm{FeCl}_{2}$ or $\mathrm{MgCl}_{2} . \mathrm{CH}_{3} \mathrm{Cl}$ was itself looked for at $3 \mu \mathrm{m}$ to test this hypothesis, but it too was not detected. This last result is not a significant test, however, because the maximum predicted intensities for the $\mathrm{CH}_{3} \mathrm{Cl}$ absorption lines were still below the detection limit.

$\mathrm{CH}_{2}$

The methylene radical, $\mathrm{CH}_{2}$, is a UV-photodissociation product of methane, and may be produced in detectable quantities in the outer regions of the IRC +10216 circumstellar envelope. A recent search for $\mathrm{CH}_{2}$ lines in the $\nu_{2}$ band near $10 \mu \mathrm{m}$, however, failed to produce any positive results (Goldhaber, Betz, and Ottusch 1986). An upper limit of $4 \times 10^{15} \mathrm{~cm}^{-2}$ was estimated for the column density by assuming a rotational temperature of $20 \mathrm{~K}$. Although this observational 
limit is more than two orders of magnitude higher than the abundance predicted from the photodissociation of methane, it confirms that $\mathrm{CH}_{2}$ is not otherwise very abundant in the higher density regions of the circumstellar cloud.

\section{Future Prospects}

The number of undetected "simple" molecules composed of $\mathrm{C}, \mathrm{N}$, and $\mathrm{O}$ is small. Other than the molecules mentioned above, the two major undetected species are $\mathrm{H}_{2} \mathrm{O}$ and $\mathrm{CO}_{2}$. Although both molecules should be easily detectable in oxygen-rich circumstellar shells, neither species can be observed in the infrared because of strong absorption from its telluric counterpart. $\mathrm{H}_{2} \mathrm{O}$ is, of course, known from its microwave maser emission to be quite abundant in oxygen-rich supergiant stars. It may also be possible to detect infrared emission from $\mathrm{H}_{2} \mathrm{O}$ with high altitude airborne observations. Tsuji (1978) has discussed the possibility that the emission already seen at 5-8 $\mu \mathrm{m}$ in some late-type stars may be produced by blended lines of the $\nu_{2}$ band of $\mathrm{H}_{2} \mathrm{O}$. For $\mathrm{CO}_{2}$ a similar possibility holds. In oxygen-rich stars with moderate to high $\mathrm{CO}_{2}$ column densities, the $4.3 \mu \mathrm{m}$ continuum radiation that pumps the $\nu_{3}$ band of $\mathrm{CO}_{2}$ will be highly trapped. This can lead to emission in the $\nu_{3}-\nu_{1}$ band at $10.5 \mu \mathrm{m}$ which can be observed from the ground.

Two less abundant species that will undoubtedly be searched for soon are the saturated hydrides: ethane $\left(\mathrm{C}_{2} \mathrm{H}_{6}\right)$ and hydrazine $\left(\mathrm{N}_{2} \mathrm{H}_{4}\right)$. Ethane has a strong $\nu_{9}$ band at $12 \mu \mathrm{m}$, and hydrazine has a number of intense $\nu_{12}$ subbands in the $10.5 \mu \mathrm{m}$ region. Both these wavelength bands are accessible from the ground. Neither species is expected to be very abundant based on chemical equilibrium calculations; however, it must be noted that the observed hydrides are noticeably enhanced over their predicted abundances. For a strong source like IRC +10216 , a fractional abundance of $\sim 10^{-8}$ for either species should be detectable.

I wish to thank John Keady and Steven Ridgway for allowing use of their FTS data prior to publication, and also for many helpful discussions. I am also grateful to John Lacy his comments on the search for molecular hydrogen. Infrared heterodyne spectroscopy at UCB is supported in part by NSF grant AST-8204589 and by NASA grant NAGW-681.

\section{References}

Barnes, T.G., Beer, R., Hinkle, K.H., and Lambert, D.L. (1977), "A High-Resolution Infrared Spectrum of IRC +10216", Ap. J., 213, 71-78.

Beckwith, S. (1981), "The Implications of Molecular Hydrogen Emission", in Infrared Astronomy, Proc. IAU Symp. No. 96, eds., C.G. Wynn-Williams and D.P. Cruikshank, (D. Reidel Co.), 167-178.

Betz, A.L., McLaren, R.A., and Spears, D.L. (1979), " $\mathrm{NH}_{3}$ in IRC +10216", Ap. J. (Lett.), 229, L97-L100.

Betz, A.L., and McLaren, R.A. (1980), "Infrared Heterodyne Spectroscopy of Circumstellar Molecules", in Interstellar Molecules, Proc. IAU Symp. No. 87, ed. B.H. Andrew (D. Reidel Co.), 503-507.

Betz, A.L. (1981a), "Infrared Heterodyne Spectroscopy of Ammonia and Ethylene in Stars", in Laser Spectroscopy V, Proc. Fifth Int. Conf. Laser Spectrosc., (Springer-Verlag), 81-88.

Betz, A.L. (1981b), "Ethylene in IRC +10216", Ap. J. (Lett.), 244, L103-L105.

Betz, A.L., and Goldhaber, D.M. (1985), "Circumstellar Dynamics Observed by Infrared Heterodyne Spectroscopy", in Mass Loss from Red Giants, eds. M. Morris and B. Zuckerman (D. Reidel Co.), 83-84.

Clegg, R.E.S., Hinkle, K.H., and Lambert, D.L. (1982), "High-Resolution $3 \mu \mathrm{m}$ Spectroscopy of IRC +10216", Mon. Not. R. Astr. Soc., 201, 95-103. 
Geballe, T.R., Wollman, E.R., and Rank, D.M. (1973), "Observations of Carbon Monoxide at 4.7 Microns in IRC +10216, VY Canis Majoris, and NML Cygni", Ap. J., 183, 499-504.

Geballe, T.R., Lacy, J.H., and Beck, S.C. (1979), "The 8 Micron Band of Silicon Monoxide in the Expanding Cloud Around VY Canis Majoris", Ap. J. (Lett.), 230, L47-L51.

Goldhaber, D.M., and Betz, A.L. (1984), "Silane in IRC +10216", Ap. J. (Lett.), 279, L55-L58.

Goldhaber, D.M., Betz, A.L., and Ottusch, J.J. (1986), "New Lines of Ethylene and a Search for Methylene in IRC +10216", submitted to Ap.J.

Hall, D.N.B., and Ridgway, S.T. (1978), "Circumstellar Methane in the Infrared Spectrum of IRC +10216", Nature, 273, 281-282.

Hall, D.N.B., Ridgway, S.T., Bell, E.A., and Yarborough, J.M. (1978a), Proc. Soc. Photo-Optical Instr. Engr., 172, 121.

Hall, D.N.B., Kleinmann, S.G., Ridgway, S.T., and Gillett, F.C. (1978b), "High-Resolution 1.5-5 Micron Spectroscopy of the Becklin-Neugebauer Source in Orion", Ap.J. (Lett.), 223, L47L50.

Hinkle, K.H. (1983), "High Resolution Spectroscopy of Late-Type Circumstellar Shells", Publ. Astr. Soc. Pacific, 95, 550-555.

Huggins, P.J., Glassgold, A.E., and Morris, M. (1984), "CN and $\mathrm{C}_{2} \mathrm{H}$ in $\mathrm{IRC}+10216$ ", Ap.J., 279, 284-290.

Keady, J.J. (1982), "The Circumstellar Envelope of IRC +10216", Ph.D. Thesis, New Mexico St. Univ.

Keady, J.J. (1985), private communication.

Keady, J.J. (1986), "Molecular Hydrogen Quadrupole Transitions in IRC + 10216", preprint.

Keady, J.J., and Hinkle, K.H. (1986), in preparation.

Keady, J.J., and Ridgway, S.T. (1986), in preparation.

Keady, J.J., Hall, D.N.B., and Ridgway, S.T. (1986), in preparation.

Kostiuk, T. Espenak, F., Deming, D., Mumma, M.J., Zipoy, D., and Keady, J. (1985), "Study of Velocity Structure in IRC +10216 Using Acetylene Line Profiles", B.A.A.S., 17, 570.

Lacy, J. (1979), Ph.D. thesis, University of California, Berkeley.

Lacy, J. (1985), private communication.

McCabe, E.M., Smith, R.C., and Clegg, R.E.S. (1979), "Molecular Abundances in IRC + 10216", Nature, 281, 263-266.

McLaren, R.A., and Betz, A.L. (1980), "Infrared Observations of Circumstellar Ammonia in OH/IR Supergiants", Ap. J. (Lett.), 240, L159-L163.

Merrill, K.M., and Ridgway, S.T. (1979), "Infrared Spectroscopy of Stars", Ann. Rev. Astron. Astrophys., 17, 9-41.

Omont, A. (1985) "The Physical and Chemical Structure of Circumstellar Envelopes", in Mass Loss from Red Giants, eds. M. Morris and B. Zuckerman (D. Reidel Co.), 269-289.

Rank, D.M., Geballe, T.R., and Wollman, E.R. (1974), "Detection of ${ }^{17} \mathrm{O}$ in IRC +10216 ", Ap.J. (Lett.), 187, L111-L112.

Ridgway, S.T., Hall, D.N.B., Kleinmann, S.G., Weinberger, D.A., and Wojslaw, R.S. (1976), "Circumstellar Acetylene in the Infrared Spectrum of IRC +10216", Nature, 264, 345-346.

Ridgway, S.T., Carbon, D.F., and Hall, D.N.B. (1978), "Polyatomic Species Contributing to the Carbon-Star 3 Micron Band", Ap. J., 225, 138-147.

Ridgway, S.T., and Hall, D.N.B. (1980), "Spectroscopic Studies of IRC +10216 and Similar Objects", in Interstellar Molecules, Proc. IAU Symp. No. 87, ed. B.H. Andrew (D. Reidel Co.), 509-514. 
Ridgway, S.T., and Keady, J.J. (1981), "The Nature of IRC +10216", Phil. Trans. R. Soc. London, A303, 497-502.

Rinsland, C.P., Baldacci, A., and Rao, K.N. (1982), "Acetylene Bands Observed in Carbon Stars: A Laboratory Study and an Illustrative Example of its Application to IRC +10216", Ap. J. (Suppl.), 49, 487-513.

Schrey, U., Rothermel, H., Kaufl, H.U., and Drapatz, S. (1985), "Recent Measurements on Mars and IRC + 10216 Made with the MPE 10 Micron Heterodyne Receiver", in Proc. URSI Symp. on Millimeter and Submillimeter Wave Radio Astronomy, ed. J. Gomez-Gonzales, Granada, 11-14 Sept. 1984, pp. 213-216.

Tsuji, T. (1978), "A Possible Identification of $\mathrm{H}_{2} \mathrm{O}$ Thermal Emission in the Infrared Spectra of Late-type Stars", Astron. Astrophys., 68, L23-L26.

Wannier, P.G. (1985), "Mass Loss from Red Giants: Infrared Spectroscopy", in Mass Loss from Red Giants, eds. M. Morris and B. Zuckerman (D. Reidel Co.), 65-79.

Wootten, A., Lichten, S.M., Sahai, R., and Wannier, P.G. (1982), "CN Abundance Variations in the Shell of IRC +10216", Ap.J., 257, 151-160.

Zuckerman, B. (1980), "Envelopes Around Late-type Giant Stars", Ann. Rev. Astron. Astrophys., 18, 263-288.

\section{DISCUSSION}

GLASSGOLD: Is there a problem in determining the continuum, and deducing accurate column densities, in your measurements?

BETZ: The IF bandwidth of the $10 \mu \mathrm{m}$ heterodyne receiver and the number of filterbank channels limits our data to a velocity coverage of $26 \mathrm{~km} / \mathrm{s}$ in either infrared sideband. Generally the lines observed are less than 10 to $15 \mathrm{~km} / \mathrm{s}$ wide near the continuum level and reasonable continuum measurements can be made. Since we observe lines in absorption, an absolute measurement of intensity is not required to get good column densities. If the gas measured has a rotational temperature $>300 \mathrm{k}$, however, we consider the effect of $10 \mu \mathrm{m}$ continuum radiation from co-existing warm dust on the observed depth of the line profiles.

P.A. FELDMAN: Do you have any explanation for the relative abundance of ethylene (cf. to acetylene) which you find in IRC+10 216 ? The equilibrium "freeze-out" model for IRC $+10^{\circ} 216$ computed by Lafont et al. leads to $\left[\mathrm{C}_{2} \mathrm{H}_{4}\right] /\left[\mathrm{C}_{2} \mathrm{H}_{2}\right] \sim 10^{-5}$ to $10^{-6}$. Thus, even if the original (Ridgway et al. 1976) value of $3 \times 10^{19} \mathrm{~cm}^{-2}$ for $\mathrm{N}\left(\mathrm{C}_{2} \mathrm{H}_{2}\right)$ is re-adopted for the $12 \mu \mathrm{m}$ column density, $\mathrm{N}\left(\mathrm{C}_{2} \mathrm{H}_{4}\right)$ is not expected to exceed $\sim 3 \times 10^{14} \mathrm{~cm}^{-2}$ on the basis of equilibrium "freeze-out" chemistry. Also, permit me to mention that our poster paper on CFHT FTS observations of IRC $+10^{\circ} 216$ at $3.3 \mu \mathrm{m}$ deals, in part, with our non-detection of the $v_{11}$ fundamental band of $\mathrm{C}_{2} \mathrm{H}_{4}$. However there is apparently no discrepancy, since our $3 \sigma$ upper limit to $\mathrm{N}\left(\mathrm{C}_{2} \mathrm{H}_{4}\right)$ is a factor of 6 higher than your quoted estimate. In addition, at $12 \mu \mathrm{m}$ you see deeper into the circumstellar envelope than we do at $3 \mu \mathrm{m}$. 
BETZ: The observed abundance ot etnylene seems high only in comparison to the predictions of some "freeze-out" models. Other "freeze-out" models come closer on ethylene but fail tor other species. Regardless, it is quite evident that other mechanisms are at work in circumstellar clouds besides thermal-equilibrium chemistry.

WILLIAMS: Following Paul Feldman"s comment concerning the "freeze-out" model, it is now clear that this model is inadequate and that a rich ion-molecule chemistry occurs in the flow, the ionization being produced by the ambient radiation field. Work by e.g. Millar and Nejad have a successful model of IRC $+10^{\circ} 216$ which is based on this idea.

SAHAI: (i) Sis has its fundamental band at $13.7 \mu$ and may be a future prospect for detection in circumstellar envelopes. (ii) The problem of not knowing to what depth one is looking along the line of sight in absorption lines can be overcome by looking at resonant scattered emission from regions offset from the continuum source as done by us using 4.6 CO lines (Sahai \& Wannier, 1985).

BETZ: (i) FTS instrument on the KPNO 4-m telescope will be best for Sis. (ii) Line-of-sight absorption measurements need to know the circumstellar dust opacity at the wavelength of interest to determine the radial depth of circumstellar gas that is probed. Resonant scattering measurements are possible only on abundant species and not the trace molecules that are of interest here.

UNDERHILL: You noted three lines of one infrared band in IRC $+10^{\circ} 216$ pointing out that the shortward displacement of the deepest point of the strongest line (from the lowest rotational level) was greater than that of the next weaker line (from a higher level) which, in turn, was greater than that of the third. line (from the higher level). This sequence you interpreted as evidence for a velocity of outflow which increases outwards. It is not possible to make such an interpretation because of the details of radiative transfer in an expanding atmosphere. In an expanding atmosphere, the Eddington-Barbier relation is no longer valid and you cannot identify the deepest point in an absorption line with a particular geometric level in the atmosphere. The most you may conclude is that the terminal velocity exceeds the velocity indicated by the shortward displacement of the core of the strongest line. These statements become clear when you plot the constant-velocity surfaces in the atmosphere which you are modelling and calculate what is the contribution of each part of the surface to the line profile.

BETZ: The optically thin lines of ammonia that we see are formed in circumstellar gas $\sim 10^{15}$ to $10^{16} \mathrm{~cm}$ from the star. The illustration shows that cooler gas observed in the $\mathrm{aR}(0,0)$ line has a slightly higher expansion velocity on the average compared to warmer gas seen in the $\mathrm{aQ}(3,2)$ line. Lines of differing excitation preferentially probe different regions of the expanding and cooling circumstellar cloud. 
LAMBERT: It is not quite true to say that there are no optical observations. About 20 years ago, Herbig identified SCO around 6000A in emission in the circumstellar shell of VY CMa. I have subsequently identified vo and Tio quite readily. I assume that these lines are excited by fluorescence. The fact that their rotational temperature is $\sim 600^{\circ} \mathrm{K}$ proves their circumstellar origin. 